\title{
Measurements of neutrino-nucleus scattering
}

\section{Wilkinson*}

University of Bern, Albert Einstein Center for Fundamental Physics, Laboratory for High Energy Physics (LHEP), Bern, Switzerland

E-mail: callum.wilkinson@lhep.unibe.ch

Current and planned neutrino oscillation experiments operate in the 0.1-10 GeV energy regime. At these energies, the neutrino cross section is not well understood: a variety of interaction processes are possible and nuclear effects play a significant role. Here, the conceptual problems that affect measuring and understanding neutrino cross sections are introduced, and the status of neutrino cross section measurements for $\mathrm{CC} 0 \pi$ and $\mathrm{CC} 1 \pi$ channels are discussed.

Neutrino Oscillation Workshop (NOW2018)

9-16 September, 2018

Rosa Marina (Ostuni, Brindisi, Italy)

* Speaker. 


\section{Introduction}

Neutrino oscillations are a well-established phenomenon, which depend on the neutrino energy, $E_{v}$, and distance travelled. A number of current and planned accelerator neutrino experiments aim to make precise measurements of the underlying parameters which govern the oscillation probability. These all operate in the few-GeV energy region, in the so-called "transition region", where multiple neutrino interaction modes contribute, as shown in Figure 1.

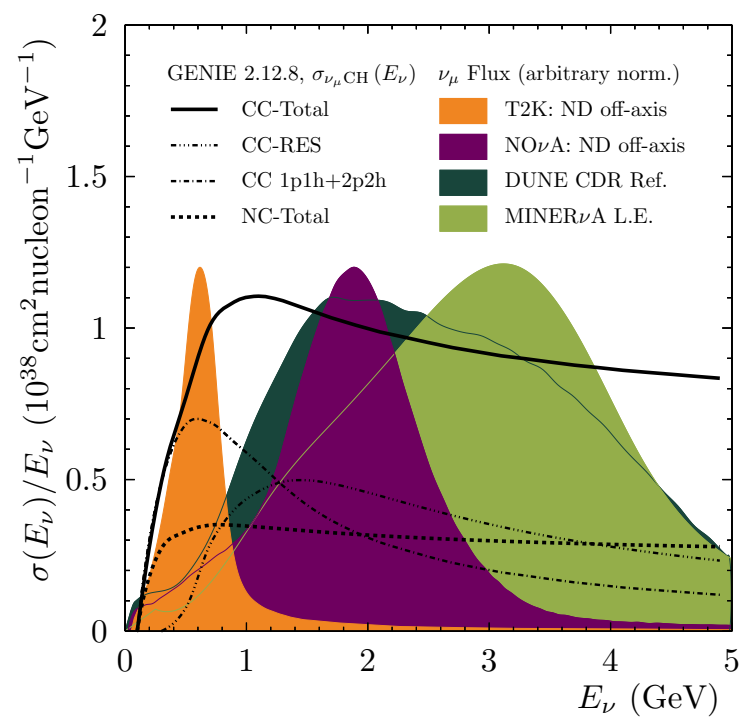

Figure 1: Neutrino fluxes, as a function of energy, of current and future accelerator-based neutrino experiments. The fluxes relevant to neutrino interaction measurements are shown, from the T2K experiment off-axis near detector (ND) [1], NOvA ND [2], and MINERvA (Low Energy configuration only [3]). One future program, DUNE [4], is shown; the HK flux is very similar to the T2K ND off-axis flux. Also overlaid are the total CC cross section divided by energy, according to GENIE 2.12.8 [5]. Figure reproduced from Ref. [6].

The basic method for conducting an accelerator neutrino oscillation analysis is to compare the rates at a near detector, located close to the production point before oscillations can occur, and at a far detector, located near the oscillation maximum. The rate in each detector, $R(\overrightarrow{\mathbf{x}})$, as a function of reconstructed final-state particle kinematic quantities, $\overrightarrow{\mathbf{x}}$, can be expressed as:

$$
R(\overrightarrow{\mathbf{x}})=\int_{E_{\min }^{E_{\max }} \Phi\left(E_{v}\right) \times \sigma\left(E_{v}, \overrightarrow{\mathbf{x}}\right) \times \varepsilon(\overrightarrow{\mathbf{x}}) \times P\left(v_{A} \rightarrow v_{B}\right),}^{\text {Near detector }}
$$

where $\Phi\left(E_{v}\right)$ is the neutrino flux as a function of $E_{v}, \sigma\left(E_{v}, \overrightarrow{\mathbf{x}}\right)$ is the cross section, $\varepsilon(\overrightarrow{\mathbf{x}})$ is the detector efficiency, and $P\left(v_{A} \rightarrow v_{B}\right)$ is the oscillation probability. It is clear that a good understanding of the neutrino cross section, $\sigma\left(E_{v}, \overrightarrow{\mathbf{x}}\right)$, is critical for carrying out an oscillation analysis, as it relates the neutrino energy with the variables that can be measured in the detector. Although the near detector is able to make in situ measurements of the flux, cross section and detector efficiency, which constrain uncertainties before convolving the oscillation probability at the far detector, simply taking a near/far ratio does not fully cancel systematics because there is a dramatic change in the neutrino energy and flavor distribution over which the integral runs between the detectors.

\section{Neutrino cross section concepts}

A breakdown of the different major contributions to the neutrino-nucleus cross-section in the few-GeV region is indicated in Figure 1. The contributions, or modes, can be conceptually separated in terms of the energy transfer to the struck nucleus. At low energy transfers, the interaction is with the nucleus as a whole, either elastically, or through the excitation of a giant nuclear resonance. At intermediate energy transfers, the interaction is with the nucleon, first as quasi-elastic scattering, or single nucleon knock-out $(1 \mathrm{p} 1 \mathrm{~h}),\left(\vec{v}_{l}^{\prime}+n(p) \rightarrow l^{-(+)}+p(n)\right.$, and then at higher energy transfers, through the excitation of a nucleon resonance (RES), which decays to produce a pion and a nucleon ${ }^{1}$. At large energy transfers, the interaction is with a constituent parton inside the nucleon,

\footnotetext{
${ }^{1}$ Note that in rare cases, nucleon resonances can decay to final states which include multiple pions, or heavier mesons, such as kaons [7].
} 
in deep inelastic scattering (DIS). Because the nucleons are bound within the nucleus, when the interaction is with a nucleon (1p1h, RES), the response depends on the details of the initial nuclear state, in particular the initial state nucleon momentum distribution, and the energy required to liberate a bound nucleon. Additionally, interactions with more than one nucleon are possible, generally referred to as multinucleon knock-out $(2 \mathrm{p} 2 \mathrm{~h})$. The transition between interactions with an entire nucleus, to interactions with a nucleon, to interactions with a parton, is a significant challenge to building a consistent cross-section model.

Critically, because the neutrino energy and energy transfer to the nucleus cannot be reconstructed on an event-by-event basis, it is necessary to have a good understanding of the entire nuclear response, as experiments have to implicitly integrate over the energy transfer when measuring an event rate (Equation 1.1). Worse, because neutrinos can interact anywhere inside the nucleus, hadrons are produced deep inside the nuclear medium, and have a high probability of interacting before they escape, and are visible in the detector. These interactions, which modify the final-state particle content, are referred to as final state interactions (FSI). As a result, we cannot measure interaction modes such as $1 \mathrm{p} 1 \mathrm{~h}, 2 \mathrm{p} 2 \mathrm{~h}$ or RES interactions unambiguously. Instead, we measure final state topologies, which are defined by the observable final-state particle content. For example, $\mathrm{CC} 0 \pi$, where a single muon is observed, and no pions (or, generally, any other mesons). $\mathrm{CC} 0 \pi$ may be dominated by $1 \mathrm{p} 1 \mathrm{~h}$ and $2 \mathrm{p} 2 \mathrm{~h}$ processes, but RES processes may also contribute if the pion produced at the vertex is absorbed through FSI.

Measurements of interaction topologies do not correct for FSI, and are therefore more modelindependent than measurements of interaction modes where the experiment has imposed assumptions about FSI. However, it is a significant challenge to use measurements of interaction topologies to constrain the underlying cross-section model parameters and reduce the systematic uncertainties for oscillation experiments [8]. Different components of the full neutrino-nucleus scattering model can be constrained through other sources to make the problem more tractable. Electron-nucleus scattering data can be used to constrain nuclear model uncertainties, as the nucleus is the same in both cases, and pion-nucleus scattering data can be used to constrain the effects of FSI to some extent (see, for example, Ref. [9]).

\section{3. $\mathrm{CCO} \pi$ status}

Much of the theoretical work on neutrino-nucleus cross-section models over the last 10 years has been motivated by observed discrepancies between old models and MiniBooNE CCO $\pi$ data $[10$, 11]. The introduction of nuclear effects such as $2 \mathrm{p} 2 \mathrm{~h}$, and a more sophisticated treatment of the initial nuclear state has been successful in qualitatively describing that data, and agrees with electron scattering data. However, attempts to confront the models with all available data have shown that some issues remain $[12,13,14]$.

There have been a number of recent measurements of $\mathrm{CC} 0 \pi$ which aim to test the new theoretical models, and offer new data to help refine them. Both T2K [15, 16] and MINERvA [17, 18, 19] have made measurements as a function of outgoing muon kinematics which show broad agreement with the available $\mathrm{CC} 0 \pi$ models, but are not very sensitive to nuclear effects, and lack power to differentiate between competing models, although certain tricks can be used to improve the sensitivity [20]. Additional variables, based on momentum imbalances have been proposed [21], and measured, again by both T2K [22] and MINERvA [23]. These measurements also require the reconstruction of a proton in the final state, and appear to be a powerful new tool for differentiating between models [24]. However, it is likely that more theoretical work will be needed to take full advantage of these new datasets.

Additionally, CC-inclusive datasets from MINERvA [25, 26] which focus on low-momentum transfer kinematics offer a new probe of the region where multi-nucleon, and other nuclear effects, have a strong effect.

\section{4. $\operatorname{CC1} \pi$ status}

$\mathrm{CC} 1 \pi$ cross sections are more difficult to model than $\mathrm{CC} 0 \pi$ cross sections as they contain significant contributions from different energy transfer regimes: coherent pion production, RES, 
and DIS can all produce a muon and a single pion in the final state ${ }^{2}$. They are also more difficult to measure, as they contain higher multiplicity events, making a model-independent cross section more difficult to extract [6]. Attempts to compare cross-section models to CC1 $\pi$ neutrino-nucleon data have shown that a consistent nucleon-level model is possible [32, 33, 34], but tension has been observed between the neutrino-nucleon data and neutrino-nucleus data [35].

A comparison between all recent neutrino-nucleus $\mathrm{CC} 1 \pi$ data is given in Ref. [6], which shows that the measured muon kinematic variables broadly agree with a reference GENIE v2.12.8 model [5], but that the measured pion kinematic variables do not, although there is some consistency between diverse datasets. This lack of overall agreement is representative of all generator models currently available. The currently available neutrino-nucleus pion-production models lack predictive power, a potentially serious issue for oscillation measurements.

\section{Concluding remarks}

Measuring neutrino cross sections, and then using those measurements to constrain a full cross section model suitable for accelerator neutrino oscillation experiments, are both significant challenges. There has been a theoretical focus on $\mathrm{CC} 0 \pi$ cross section modelling over the last 10 years, which has been complemented with an experimental program to test those models. Recent, more stringent probes from the experimental community (measurements of transverse imbalances) will motivate further work to refine the theoretical models. Overall, the relationship between experiment and theory is good news for the $\mathrm{T} 2 \mathrm{~K}$ and Hyper-K experiments, which use $\mathrm{CC} 0 \pi$ as a signal process. $\mathrm{CC} 1 \pi$ and higher invariant mass process, are in general less well understood theoretically, without the same level of recent theory engagement as CCO $\pi$. Similarly, the measurements of CC1 $\pi$ do not agree well, and indicate tension between channels and between experiments. This is a less positive picture for NOvA and DUNE, for which $\mathrm{CC} 1 \pi$ is a signal process, particularly because the improvements in $\mathrm{CC} 0 \pi$ modelling and measurements took many years to achieve. A final comment, relevant for DUNE, is that much of the experimental data is on hydrocarbon, or water targets, and as such, the atomic number dependence of the cross section models we have is not well tested. This will be partially mitigated by the SBN program at Fermilab, but not for the broad range of energy transfer which will be relevant for DUNE.

\section{References}

[1] T2K collaboration, T2K neutrino flux prediction, Phys. Rev. D87 (2013) 012001 [1211. 0469 ].

[2] NOvA collaboration, NOvA flux prediction, .

[3] MINERvA collaboration, Neutrino Flux Predictions for the NuMI Beam, Phys. Rev. D94 (2016) 092005 [1607.00704].

[4] DUNE collaboration, Long-Baseline Neutrino Facility (LBNF) and Deep Underground Neutrino Experiment (DUNE), 1512.06148.

[5] C. Andreopoulos et al., The GENIE Neutrino Monte Carlo Generator, Nucl. Instrum. Meth. A614 (2010) 87 [0905.2517].

[6] K. Mahn, C. Marshall and C. Wilkinson, Progress in Measurements of 0.1-10 GeV Neutrino-Nucleus Scattering and Anticipated Results from Future Experiments, Ann. Rev. Nucl. Part. Sci. 68 (2018) 105 [1803.08848].

[7] Particle Data Group collaboration, Review of particle physics, Phys. Rev. D 98 (2018) 030001.

[8] P. Stowell et al., NUISANCE: a neutrino cross-section generator tuning and comparison framework, JINST 12 (2017) P01016 [1612.07393].

[9] E. S. Pinzon Guerra et al., Using world charged $\pi^{ \pm}$-nucleus scattering data to constrain an intranuclear cascade model, 1812.06912.

[10] MiniBooNE collaboration, First measurement of the muon neutrino charged current quasielastic double-differential cross section, Phys. Rev. D81 (2010) 092005 [1 002 . 2680].

\footnotetext{
${ }^{2}$ Measurements of coherent pion production have been carried out, and can be used to independently constrain that part of the model $[27,28,29,30,31]$.
} 
[11] MINIBooNE collaboration, First measurement of the muon antineutrino double-differential charged current quasi-elastic cross section, Phys. Rev. D88 (2013) 032001 [1301. 7067 ].

[12] C. Wilkinson, Constraining neutrino interaction uncertainties for oscillation experiments, $\mathrm{Ph} . \mathrm{D}$. thesis, Sheffield U., 2015-06-12.

[13] C. Wilkinson et al., Testing charged current quasi-elastic and multinucleon interaction models in the NEUT neutrino interaction generator with published datasets from the MiniBooNE and MINERvA experiments, Phys. Rev. D93 (2016) 072010 [1601. 05592].

[14] M. Betancourt et al., Comparisons and Challenges of Modern Neutrino Scattering Experiments (TENSIONS2016 Report), Phys. Rept. 773-774 (2018) 1 [1805.07378].

[15] T2K collaboration, Measurement of double-differential muon neutrino charged-current interactions on $\mathrm{C}_{8} \mathrm{H}_{8}$ without pions in the final state using the T2K off-axis beam, Phys. Rev. D93 (2016) 112012 [1602.03652].

[16] T2K collaboration, First measurement of the $v_{\mu}$ charged-current cross section on a water target without pions in the final state, Phys. Rev. D97 (2018) 012001 [1708.06771].

[17] MINERVA collaboration, Measurement of electron neutrino quasielastic and quasielasticlike scattering on hydrocarbon at $\left\langle E_{v}\right\rangle=3.6$ GeV, Phys. Rev. Lett. 116 (2016) 081802 [1509. 05729 ].

[18] MINERVA collaboration, Measurement of the muon anti-neutrino double-differential cross section for quasi-elastic scattering on hydrocarbon at $E_{v} \sim 3.5$ GeV, Phys. Rev. D97 (2018) 052002 [1801.01197].

[19] MINERVA collaboration, Measurement of Quasielastic-Like Neutrino Scattering at $\left\langle E_{v}\right\rangle \sim 3.5 \mathrm{GeV}$ on a Hydrocarbon Target, Phys. Rev. D99 (2019) 012004 [1811. 02774].

[20] C. Wilkinson and K. S. McFarland, Direct extraction of nuclear effects in quasielastic scattering on carbon, Phys. Rev. D94 (2016) 013013 [1602 . 07096].

[21] X. G. Lu et al., Measurement of nuclear effects in neutrino interactions with minimal dependence on neutrino energy, Phys. Rev. C94 (2016) 015503 [1512.05748].

[22] T2K collaboration, Characterisation of nuclear effects in muon-neutrino scattering on hydrocarbon with a measurement of final-state kinematics and correlations in charged-current pionless interactions at T2K, Phys. Rev. D98 (2018) 032003 [1802.05078].

[23] MINERvA collaboration, Measurement of final-state correlations in neutrino muon-proton mesonless production on hydrocarbon at $\left\langle E_{V}\right\rangle=3 \mathrm{GeV}$, Phys. Rev. Lett. 121 (2018) 022504 [1805. 05486 ].

[24] S. Dolan, Exploring Nuclear Effects in Neutrino-Nucleus Interactions Using Measurements of Transverse Kinematic Imbalance from T2K and MINERvA, 1810.06043.

[25] MINERVA collaboration, Identification of nuclear effects in neutrino-carbon interactions at low three-momentum transfer, Phys. Rev. Lett. 116 (2016) 071802 [1511. 0594 4].

[26] MINERvA collaboration, Anti-neutrino charged-current reactions on scintillator with low momentum transfer, Phys. Rev. Lett. 120 (2018) 221805 [1803. 09377 ].

[27] MiniBooNE collaboration, First Observation of Coherent $\pi^{0}$ Production in Neutrino Nucleus Interactions with $E_{v}<2$ GeV, Phys. Lett. B664 (2008) 41 [0803. 3423].

[28] MINER $v$ A collaboration, Measurement of coherent production of $\pi^{ \pm}$in neutrino and antineutrino beams on carbon from $E_{v}$ of 1.5 to $20 \mathrm{GeV}$, Phys. Rev. Lett. 113 (2014) 261802 [1409. 3835 ].

[29] MINOS collaboration, Measurement of single $\pi^{0}$ production by coherent neutral-current $v \mathrm{Fe}$ interactions in the MINOS Near Detector, Phys. Rev. D94 (2016) 072006 [1608 . 05702].

[30] T2K collaboration, Measurement of coherent $\pi^{+}$production in low energy neutrino-carbon scattering, Phys. Rev. Lett. 117 (2016) 192501 [1604.04406].

[31] MINERVA collaboration, Measurement of total and differential cross sections of neutrino and antineutrino coherent $\pi^{ \pm}$production on carbon, 1711.01178.

[32] C. Wilkinson et al., Reanalysis of bubble chamber measurements of muon-neutrino induced single pion production, Phys. Rev. D90 (2014) 112017 [1411.4482].

[33] P. Rodrigues, C. Wilkinson and K. McFarland, Constraining the GENIE model of neutrino-induced single pion production using reanalyzed bubble chamber data, Eur. Phys. J. C76 (2016) 474 [1601.01888].

[34] M. Kabirnezhad, Single pion production in neutrino-nucleon interactions, Phys. Rev. D97 (2018) 013002 [1711.02403].

[35] MINERvA collaboration, Tuning the GENIE Pion Production Model with MINERvA Data, 1903.01558. 